$160 \mu_{\mathrm{A}} 1 \mathrm{~m}^{-1}$ cathode sensitivity) and a low dark current (here, $0.25 \times 10^{-9} \mathrm{~A}$ at room temperature and an overall sensitivity of $200 \mathrm{~A} \mathrm{~m}^{-1}$ ); values in parentheses are for a ' $\mathrm{C}$ ' tube (corresponding data: $>110 \mu_{\mathrm{A} \mathrm{Im}}{ }^{-1} ; 3 \times 10^{-9} \mathrm{~A}$ ). As may be seen from the table, the effect of the magnetic deflection device (shown schematically in the figure) is a reduction in the dark currents by a factor of 10-40; the noise current amplitudes thus are reduced approximately by the square root of this factor, i.e. about by $3-6$, as one should expect. For comparison, cooling of the cathode to approximately dry ice temperature reduces the dark currents by a factor of about 100, and the noise currents amplitudes for an unknown reason slightly less than $\sqrt{ } 100$, namely by about a factor of 5-8. The application of both cooling and magnetic deflection results in a net reduction of three orders of magnitude in the dark currents, and of almost two orders of magnitude for the noise amplitude. The signal current, however, was found to be almost unaffected by either cooling or the magnetic deflection device.

Although we did not make an attempt to control the photocathode temperatures between dry ice and room temperature, it was a striking fact that the noise currents of the photocathodes first decreased with decreasing temperatures, reaching a minimum value somewhere between -10 and $-50^{\circ} \mathrm{C}$, and finally rose to $2-3$ times the minimum value with temperatures close to that of dry ice. Thus, in our experiments, temperatures slightly higher than dry ice temperature, which yielded the lowest dark current noise, have been used. With a gated phototube, sometimes much larger dark currents than described here have been observed at dry ice temperatures, but we never encountered such instabilities and poorer noise figures at the 'elevated' temperatures of -50 to $-10^{\circ} \mathrm{C}$.

The data, as presented in the table, were obtained with two different tubes only. However, measurements on other 9558 type tubes and on the same tubes operated at a different voltage (between 800 and $1400 \mathrm{v}$ ) confirmed that the ratios of dark currents and dark current noise readings with and without deflection unit, or with and without cooling, are similar to the numbers given above, which thus are thought to be representative.

The calculated shot noise currents are found to be smaller than the measured ones. This indicates that the average number of 'dark current' electrons per pulse starting at the cathode is larger than one.

We wish to thank Dr. M. A. Biondi for his active interest in this study. This work was supported in part by the Army Research Office, Durham, North Carolina, U.S.A.

\section{References}

FARKAS, G., and VARGA, P., 1964, J. Sci. Instrum., 41, 704-5.

\title{
A high temperature furnace for a single crystal x-ray diffractometer
}

\author{
F. F. FOIT, JR, and D. R. PEACOR \\ Department of Geology and Mineralogy, University of Michigan, Ann Arbor, Michigan, \\ U.S.A. \\ MS. received 19th September 1966
}

\begin{abstract}
A furnace employing fiat cone diffraction geometry has been designed and constructed for the Buerger single-crystal $X$-ray diffractometer. The furnace has an estimated temperature capability of $1200-1300^{\circ} \mathrm{C}$ and a 24 -hour temperature stability of \pm 2 degc at $1000^{\circ} \mathrm{C}$. Temperature measurement is by means of a thermocouple and a power consumption-temperature curve. The device utilizes an aluminium collimator with a silica capillary crystal mount.
\end{abstract}

\section{Introduction}

Shortly after the advent of x-ray diffraction as a useful tool in the identification and lattice parameter determinations of solids, metallurgists and mineralogists began designing and utilizing various high temperature devices which enabled them to study structural transformations at high temperatures. The great bulk of research and development in the field of high temperature $\mathrm{x}$-ray technology, however, has been restricted to $\mathrm{X}$-ray powder analysis. Slow advancement in the realm of high temperature single crystal techniques has been in large part due to the lack of sophisticated instrumentation and the inherent difficulties involved, i.e. crystal mounting and diffraction geometry. Goldschmidt (1964) has prepared a complete bibliography of high temperature X-ray devices up to 1964 .

High temperature single crystal techniques can be divided into two categories: film methods and counter diffractometer methods. The former method has the advantage in that it enables one to record large portions of reciprocal space simultaneously. However, the examination must take place at one temperature and be of a duration sufficient to expose the film. Counter diffractometer methods, while only allowing the examination of single reflections, enable the observer to monitor the course of a transformation over a range of temperatures, thus giving valuable information on the rate. Counter methods also have the advantages of 
giving more accurate reflection profiles and intensity measurements.

The authors have designed and are now using in their laboratory an X-ray furnace which can be used both with a Buerger single-crystal counter diffractometer which employs Weissenberg geometry and a Weissenberg camera.

\section{Diffraction geometry}

Flat cone geometry was chosen because it requires only a narrow slit in the side of the furnace to allow exit of the diffracted rays, while other methods require a broad opening. The lack of large openings increases furnace efficiency and stability. In flat cone geometry, described by Buerger (1942), diffraction from each level takes place in a direction normal to the rotation axis of the crystal. Level selection is accomplished by adjusting the angle $\mu$ of the incident beam. However, flat cone geometry suffers from two disadvantages. It requires an angle $\mu$ approximately twice that required by equi-inclination geometry with the same radiation and thus reduces the number of levels attainable by approximately one half. This disadvantage may be offset, however, by using molybdenum radiation which has a wavelength less than half that of copper, which is commonly used with equi-inclination geometry. Flat cone geometry produces a blind region on each of the upper levels of the reciprocal lattice. However, many of the reflections which are unobservable owing to the blind region lie at such low $\gamma^{\prime}$ values that they are blocked by the furnace's beam stop. The use of molybdenum radiation also increases the amount of data available on each level.

The authors have prepared a Fortran programme for the computation of fiat cone diffractometer settings based upon the Fortran equi-inclination programme (DSET 3) of Prewitt (1960).

\section{Furnace design}

The furnace basically consists of three concentric alumina tubes surrounded by a water-cooled brass shell (figure 1).

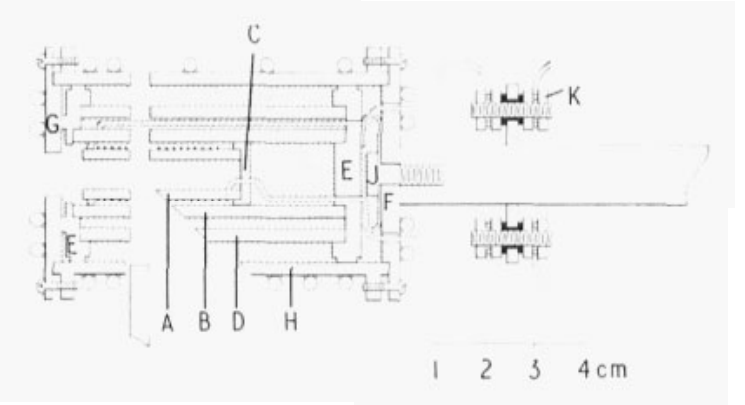

I he innermost tube or core $A$ has an inside diameter of $\frac{1}{4}$ in. and serves as a support for the winding which consists of 30 in. of 26-gauge platinum wire. The winding is covered with a thin layer of Sauereisn P-6 cement (not shown) which serves both to promote good thermal contact between the winding and the tube and to prevent possible shorting. The core is mounted inside a $\frac{1}{2}$ in. inside diameter alumina tube $B$ by means of two castings $C$ of P-6 cement. The castings are the only points of contact between the core and tube $B$; this helps to prevent heat loss from the core by conduction.

The assembly $\mathrm{A}, \mathrm{B}$ and $\mathrm{C}$ is supported inside a third alumina tube $D$ and the water-cooled shell by means of $P-6$ castings $E$. A sheet of 0.001 in. platinum foil (not shown) is placed between tubes $B$ and $D$ to act as a radiation shield.
The use of concentric alumina tubes increases the "thermal mass' of the furnace which is requisite to furnace stability and serves as insulation by reducing radiative and convective heat loss.

Clearance between the collimator support (not shown) and the furnace restricts the beam inclination angle $\mu$ to a maximum of $45^{\prime \prime}$.

When end plates $F$ and $G$ are in place, they slightly compress a leaf spring. This arrangement holds the refractory portions firmly positioned while allowing for thermal expansion. Furnace cooling is accomplished by means of $\frac{1}{8}$ in. copper tubing soft-soldered to the brass shell. The tubing connections (not shown) between the body of the shell $\mathrm{H}$ and the end plates are fittings sealed with Neoprene O-rings which are compressed by the tightening of the eight machine screws holding each end plate in place.

The furnace is supported by means of a $\frac{1}{2}$ in. steel rod firmly attached to the furnace by a single brass bolt $\mathrm{J}$. This rod also supports the bracket for the hinding posts $\mathrm{K}$. The furnace is coupled to the diffractometer by a rod and clamp assembly (figure 2) bolted to the portion of the diffracto-

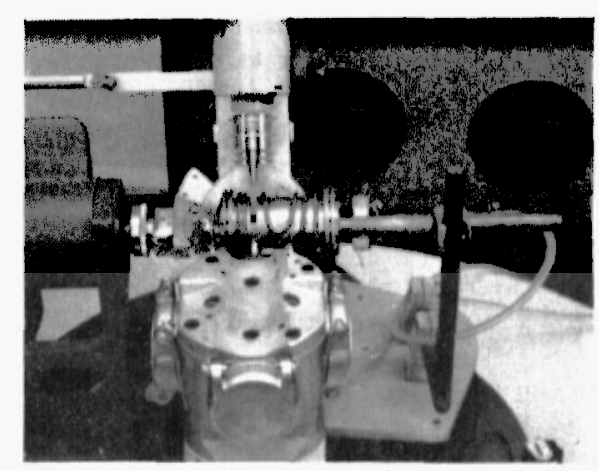

meter base which rotates for the various angles $\mu$. This arrangement allows three degrees of translational and three degrees of rotational freedom for rapid and accurate alignment of the furnace.

The furnace is designed so that with fabrication of a different supporting arrangement, the furnace can be used with a Weissenberg camera. The diffraction geometry would again be flat cone with the furnace functioning as the layer line screen and with reflections linited to the upper half of the film.

\section{Power supply}

The furnace operates on $60 \mathrm{c} / \mathrm{s}$ alternating current supplied through a $250 v_{A}$ Sola voltage stabilizing transformer with two $15 \mathrm{~A}, 2 \cdot 1 \mathrm{kVA}$ continuously variable autotransformers wired in series. One autotransformer is used as a coarse control with the other as a fine control. The system is capable of supplying $250 \mathrm{~W}$ at a maximum current of $15 \mathrm{~A}$ with a voltage stability to $\pm 1 \%$

\section{Collimation}

In intensity work, it is desirable to keep the distance between the collimator and the crystal to a minimum in order to reduce background due to air scatter. This necessitates the projection of the collimator into the furnace beyond the water-cooled shell. Because of heat transfer to the collimator, lead pinhole collimation is unsatisfactory. An aluminium collimator with a bore of $0.040 \mathrm{in}$. and a length 


\section{Notes on Experimental Technique and Apparatus}

of $2.2 \mathrm{in.}$ was therefore constructed according to the recommendation of Cid-Dresdner and Buerger (1964).

The collimator to crystal distance with this collimating system is $20 \mathrm{~mm}$ as compared with about $5 \mathrm{~mm}$ on the standard Supper diffractometer. This results in a background increase only on the zero level because of furnace blockage of air scattered radiation on the upper levels. The direct beam is stopped on the zero level by a lead beam stop (not shown) mounted on the furnace shell. On upper levels, the beam is allowed to scatter from the inside of the furnace, giving rise to a background no higher than if the beam had been allowed to impinge on a platinum beam stop mounted in the furnace core. Scattered radiation is prevented from leaving the opening for the crystal by a small lead disk mounted on the goniometer head.

\section{Crystal mounting}

Three mounting materials (water glass, Sauereisn cement and alundum cement) previously used in high temperature work were found unsatisfactory because of the occurrence of high background and powder lines. The most satisfactory method examined was that of mounting the crystal in a silica capillary. This necessitates the use of a crystal of a size $(0.2-0.3 \mathrm{~mm})$ larger than those $(0.1 \mathrm{~mm})$ normally used in inorganic work with copper radiation. Crystal absorption does not appear to be a serious problem because the mass absorption coefficients of most substances for molybdenum radiation are only a small fraction of those for copper radiation. The increased background is offset by the increased intensity of the reflections. The aluminium collimator has a measured intensity plateau in the proximity of the crystal with a diameter of $0.7 \mathrm{~mm}$, which is sufficient for the size of the crystals used.

Because of the limited amount of angular adjustment $\left(8^{\circ}\right)$ available when the crystal is inserted in the furnace, it is necessary to have the rotation axis of the crystal within a few degrees of parallel to the capillary. This is relatively simple to achieve with materials exhibiting prismatic or pseudoprismatic cleavage. In cases of materials exhibiting poor cleavage, it may be necessary to grind a sphere. Once the crystal is correctly mounted in the capillary, the capillary is trimmed to the proper length and cemented with P-6 to a short brass rod which fits a standard goniometer head.

\section{Furnace performance}

The capillary method of sample mounting places a limit on the maximum temperature at which the furnace can be used. Since silica rapidly devitrifies at temperatures above $1000^{\circ} \mathrm{C}$, only very short term experiments can be carried out above this temperature, even though the furnace possesses an estimated capability of $1200-1300^{\circ} \mathrm{C}$ (the limit is defined by vaporization of the platinum coils).

During operation of the furnace, the exit slit is covered with a strip of aluminium foil which greatly reduces furnace temperature fluctuations due to draught.
Temperature measurement is by means of both a temperature-power consumption curve and a $\mathrm{Pt}-\mathrm{Pt} / 13 \% \mathrm{Rh}$ thermocouple mounted within $2 \mathrm{~mm}$ of the specimen. The curve was constructed by making temperature measurements with the thermocouple mounted on a goniometer head and centred in the X-ray beam.

Measurements of the thermal gradient at $826^{\circ} \mathrm{C}$ show a radial region approximately $2 \mathrm{~mm} \times 3 \mathrm{~mm}$ in dimensions, slightly asymmetric with respect to the centre of the furnace, in which the temperature gradient is $5 \mathrm{degc} \mathrm{mm}^{-1}$. This area corresponds to approximately five times the diameter of the largest crystals ordinarily used in intensity work. Since the temperature gradient is greatest in the portion of the furnace below the crystal, the asymmetry could probably be eliminated by cutting a slit below the crystal which corresponds to the slit for diffraction. The furnace has a power consumption of $116 \mathrm{w}$ and a thermal stability to \pm 2 degc at $1000^{\circ} \mathrm{C}$ over a period of 24 hours. The crystal temperature, therefore, can be maintained to within $5 \mathrm{degc}$ of a desired temperature. Heating and cooling rates have been restricted to approximately $40^{\circ}$ per min because of the low resistence of alumina to thermal shock. The furnace comes to temperature equilibrium approximately 20 minutes after changing the power input.

A prismatic cleavage fragment of anorthite examined over the temperature range $25-1050^{\circ} \mathrm{C}$ showed a total angular misorientation of 30 minutes which was easily corrected using a simple counter orienting technique. The anorthite work is to be published elsewhere. This instrument will be used to investigate high temperature relations in the plagioclase feldspar system, as a continuation of this exploratory work.

\section{Acknowledgments}

The authors are indebted to the Owens-Illinois Technical Center, Toledo, Ohio, for its generous financial support of this project. Dr. Wayne Dollase of the Crystallographic Laboratory, M.I.T., and Dr. R. M. Denning of the Mineralogical Laboratory, University of Michigan, helped by discussing the problems involved in high temperature furnace construction. Dr. R. M. Denning kindly reviewed the manuscript.

\section{References}

Butrger, M. J., 1942, X-ray Crystallography (New York: Wiley).

Cid-Dresdner, H., and Buerger, M. J., 1964, J. Sc. Instrum., 41, 689 .

GoLDSCHMIDT, H. J., 1964, Bibliography 1: High-temperature $X$-ray diffraction techniques (Utrecht: International Union of Crystallography, Commission on Crystallographic Apparatus).

PREWITT, C. T., 1960, Z. Kristallogr., 114, 355-60. 